\title{
La plaza laboral como espacio de legitimación del sociólogo. Apuntes para el caso chileno
}

\author{
Dr. Nicolás Gómez Núñez*
}

\begin{abstract}
Resumen
Lo que se encuentra bajo análisis es la estructura posible de las relaciones sociales que logran dotar de poder material y simbólico a los mecanismos de inclusión del sociólogo en el Mercado de Trabajo.

El artículo fue presentado en el II Workshop sobre Dependencia Académica: "El desafío de construir Ciencias Sociales autónomas en el Sur", organizado por la Universidad Nacional de Cuyo de la ciudad de Mendoza en Argentina, el Programa Sur-Sur del Consejo Latinoamericano de Ciencias Sociales, SEPHIS, PIDAAL y SRIIRU, y que se realizó entre el 3 y 6 de noviembre de 2010.
\end{abstract}

Palabras clave: Identidad Profesional, Mecanismos de Legitimación y Mercado Laboral

\begin{abstract}
What is under analysis here is the possible structure of social relationships that provide material and symbolic power to inclusive mechanisms of sociologists within the Labour Market.

The article was presented at the Second Workshop on Academic dependence: "The challenge of building autonomous Social Sciences in the South", organized by the Universidad Nacional de Cuyo in Mendoza in Argentina, the South-South Program of the Latin American Council of Social Sciences, SEPHIS, PIDAAL and SRIIRU, which was conducted between the 3rd and 6th of November, 2010.
\end{abstract}

Key words: Professional identity, Legitimating mechanisms and Labor Market

* Escuela de Sociología, Universidad Católica Silva Henríquez, Santiago, Chile. Contacto: ngomezn@ucsh.cl 


\section{Introducción}

Este documento resume las tensiones que han sido tratadas en la etapa de construcción de un marco conceptual para abordar la identidad profesional de los sociólogos en sus desempeños laborales en Chile. El ejercicio ha sido parte de un estudio realizado entre 2010 y 2011, desde la Escuela de Sociología de la Universidad Católica Silva Henríquez (UCSH) y con el financiamiento de la Dirección de Investigación y Postgrado de la misma casa de estudio.

El surgimiento de la reflexión que a continuación se plantea responde a dos aspectos. Primero, es la continuidad de las búsquedas emprendidas, a fines de la década de los noventas, por el Dr. Justino Gómez y el Dr. Mario Sandoval sobre la Sociología del trabajo y de las profesiones, y cuyos avances han permitido concretar el interés de la UCSH por impartir la carrera de Sociología, la que a la fecha del presente documento reporta ochenta y un (81) sociólogos titulados.

La investigación en curso, ahora con un equipo que mantiene al Dr. Justino Gómez e incorpora a los licenciados Lester Reyes, Felipe Arancibia y quien escribe, retoma los planteamientos conceptuales usados por Gómez y Sandoval (2004), varias de sus opiniones críticas cuando conocen las distintas formaciones de sociólogos en Chile y en la Universidad de Lovaina en Bélgica, y concreta la indagación en la identidad profesional del sociólogo tomando como caso de estudio a los formados en la UCSH, para saber cómo ellos gestionan su identidad profesional en las plazas que el Mercado de Trabajo chileno ofrece.

El segundo aspecto. El argumento central de estas meditaciones es una respuesta a la inquietud manifestada por la Agencia que llevó a cabo el proceso de acreditación de la carrera de Sociología entre 2008 y 2009, la cual nos señaló que el cuerpo docente en particular y los distintos estamentos de la UCSH en general, no tienen conocimiento objetivo que permita saber qué hacen los sociólogos y sociólogas UCSH en el Mercado de Trabajo. 
Desde este ángulo el resultado de la presente reflexión, y de la investigación empírica que aún no finaliza, es una carta de un juego que es posible componer cuando se recorren las trayectorias de las variables que instituyen los mecanismos de legitimación de la Sociología en Chile.

Entonces, lo que se encuentra bajo análisis es la estructura posible de las relaciones sociales que logran dotar de poder material y simbólico a los mecanismos de inclusión del sociólogo en el Mercado de Trabajo. Así, la pretensión de la investigación es dar cuenta de la experiencia laboral desde los hitos que el o la socióloga usa para legitimar su desempeño profesional y para reproducir los mecanismos de legitimación inherentes al campo laboral.

Sin embargo, este recorrido no podrá ser expuesto aquí porque aún falta la etapa empírica de la investigación, y porque "el desafío de construir Ciencias Sociales autónomas en el Sur", la consigna del II Workshop sobre Dependencia Académica, organizado por la Universidad Nacional de Cuyo de la ciudad de Mendoza en Argentina, el Programa Sur-Sur del Consejo Latinoamericano de Ciencias Sociales, SEPHIS, PIDAAL y SRIIRU, requiere una discusión que emerja desde la historicidad desplegada por los practicantes de las Ciencias Sociales, o, como lo veremos a continuación, desde la descripción de la trayectoria de las variables que construyen sus plazas laborales.

Esta entrada al tópico del II Workshop sobre Dependencia Académica supone, por un lado, que la formación en Sociología se encuentra tensionada por la calidad de las demandas de la plaza laboral que se ofrece en el Mercado de Trabajo. En consecuencia, los objetos de investigación de la Sociología en particular y de las Ciencias Sociales en general, experimentan variaciones que develan la transformación en la matriz disciplinaria.

Si bien estas tensiones y variaciones son regulares en el despliegue de las Ciencias Sociales, en este documento proponemos que nos encontramos en una etapa distinta a la que se observó en el período que va desde 1950 a 1970, especialmente porque hay 
contenidos diferentes en la socialización del sociólogo en su plaza laboral, permitiendo que se organicen identidades profesionales adecuadas a la forma de estar en el Mercado de Trabajo.

Por otro lado, al indicar que es el contenido denso de la socialización en la plaza laboral el factor que determina la identidad de la Sociología, al menos en Chile desde 1990 hasta nuestros días, estamos suponiendo que la autonomía de las Ciencias Sociales en países como el nuestro, se comprende si definimos a la autonomía como la interdependencia de las formas de producir y distribuir conocimiento en un sistema de relaciones del campo laboral que, a continuación, nombramos como la plaza laboral.

Así, el obrar académico no puede definirse como dotado con la capacidad de gobernar su desempeño, debido a que los modelos de investigación, las prácticas de transmisión de los saberes, las redes de los practicantes y sus plazas laborales, se encontrarían instituidas por ellos y por los no científicos o no académicos, los cuales se vuelven inherentes al desempeño científico y profesional.

La confirmación de este supuesto, el de que los contenidos de las plazas laborales son los que permiten la identidad profesional y dan legitimidad al desempeño de la Sociología, explicaría tres asuntos.

El primero se refiere a que la dependencia de la identidad profesional del sociólogo con los contenidos de la plaza laboral, devalúa modelos de identidad profesional. El segundo asunto se refiere a que la adopción de los procedimientos de estandarización de los contenidos que se imparten mediante la carrera de Sociología, responde a los nuevos mecanismos de legitimación que han logrado reproducir la matriz disciplinaria, y cuya expresión es la acreditación de las competencias y de los procesos que producen a un profesional.

Entonces, la acreditación es un mecanismo que desdiferencia lo que antaño se vivía como el campo disciplinario, el cual esta- 
ba simbólicamente radicado en las relaciones de los practicantes que se reconocían y eran reconocidos como los sociólogos. Por tanto, la acreditación es un indicador de la nueva matriz disciplinaria porque instituye distinciones: hay formación de sociólogos en virtud de la ecumene epistémica que se co-construye por los integrantes enrolados a un proceder pero cuya procedencia es diversa y dispersa.

Aquí estarían las carreras de sociología acreditadas por la Comisión Nacional de Acreditación (www.cnachile.cl), y otras formaciones de sociólogos que no logrando este sello de la matriz disciplinaria, develarían su imposibilidad para arribar al contemporáneo ethos ordenador, o se ven impedidas de establecer una co-gestión de los contenidos de formación de sociólogos según la dependencia que tiene la docencia y la investigación con el Mercado de Trabajo. Aquí estarían las carreras de sociología no acreditadas por la Comisión Nacional de Acreditación y las que se acercan o alejan del mecanismo desdiferenciador mediante elucubraciones que se expresan discursivamente y que se construyen colectivamente por los que son la comunidad sociológica de la universidad.

El tercer y último asunto es aplicable a las comunidades de sociólogos que desenvuelven la Sociología con la mirada puesta en la calidad de la plaza laboral ofrecida por el Mercado de Trabajo, el cual se articula entre el Estado, organizaciones con fines de lucro y organizaciones sin fines de lucro. Y permite comprender por qué, al interior de los procedimientos de la formación de sociólogos, hay un énfasis en el contenido del vínculo entre el docente y el alumno.

Esto, porque en ese contenido se transfiere la información social y técnica que le permite al nuevo sociólogo gestionar el "conocimiento tácito" (Polanyi, Apud. Kuhn, 1996:292) de la plaza laboral posible, y desde donde se define como sociólogo. 


\section{La construcción social de la identidad disciplinaria}

A continuación describiremos los contenidos que el sociólogo tiene a disposición para reproducir los mecanismos de legitimación que se presentan como inherentes al campo laboral. Para desplegar este asunto, dibujamos la construcción social de la identidad disciplinaria poniendo énfasis en dos variables: la trayectoria de la plaza laboral y los contenidos que sus ocupantes deben usar.

\section{1. a. El origen del prestigio de la Sociología en Chile}

La institucionalización de la Sociología en Chile es un proceso que vincula el quehacer propiamente académico universitario con el desempeño de la política pública en el Estado, donde los contenidos de sus vinculaciones estuvieron centrados en el estudio del desarrollo y en las formas para lograr recursos materiales y simbólicos desde los circuitos de legitimación internacional.

En el despliegue de este proceso se observa la inauguración de grupos de investigación con sociólogos que logran sus grados académicos fuera de Chile, realizan investigación empírica desde el método cuantitativo, miran lo social desde la teoría funcionalista (Brunner, 1988:231) y se asumen como destinados a producir un saber eficiente en la entrega de soluciones prácticas a la modernización.

Esta Sociología tuvo una existencia exclusiva, entre 1957 a 1967, en tres organizaciones: Instituto de Sociología de la Universidad de Chile, Facultad Latinoamericana de Ciencias Sociales y Escuela de Sociología de la Universidad Católica.

El contenido denso de estas primeras plazas laborales reporta inmediatamente un prestigio privado público que inviste al individuo con varias posesiones simbólicas como, por ejemplo: el mismo nombre de sociólogo y la plaza laboral que le permite estar en los equipos profesionales y de investigación que administran la política pública. 
Entonces, el Estado fue un sistema de legitimación de la Sociología porque dio cabida a estos nuevos funcionarios calificados para que establecieran los procedimientos científicos que hacían probable la figuración del futuro deseado para Chile, a saber: el de ser un país desarrollado, a través de un conjunto de herramientas y técnicas de la Sociología que permitirían gobernar las condiciones existentes en lo social para modificar el futuro probable, a saber: el que Chile siguiera siendo un país subdesarrollado.

Este proceso ha sido interpretado como una rivalidad entre la Sociología de Cátedra o ensayística y la Sociología Científica o Profesional, debido a que se podría observar la llegada de unos practicantes con formación sociológica que establecen una competencia basada en la promoción de una Ciencia Social empírica en contra de los que habían incorporado a sus contenidos de cátedra, los trabajos de Sociología que descubrieron en razón de sus preocupaciones por la transformación de la sociedad chilena.

Según Beigel (2010), esta interpretación dicotómica impide observar las determinantes estructurales y las prácticas sociales. Por ejemplo, al cuestionar esta lógica se constata que nombrar la división entre las dos Sociologías es un mito porque todos los habitantes de esa naciente estructura ocupacional eran "sociólogos de cátedra" que: "pretendían transformarse en profesores full time", "dirigir las nacientes escuelas", captar los recursos de la cooperación internacional (Beigel, 2010:32), buscaban su legitimación en el circuito regional y su disputa por las plazas laborales del mercado de posiciones académicas estaba guiado por los dividendos de la internalización, entre ellos: los fondos para la asistencia técnica (Beigel, 2010:37).

Luego, entre 1960 y 1973, el mercado laboral está constituido por la coexistencia de un sector "tecno-burocrático" y otro de “posiciones académicas" (Brunner, 1988:231-234), los cuales permiten que el sociólogo se legitime por sus desempeños como investigador, especialmente en los asuntos de la economía agraria y la pobreza; y en las distintas fases de la planificación. 
En ese contexto Barrios observa que hay un tránsito en el desempeño profesional que es atribuible, por un lado, a los contenidos cada vez más especializados de las plazas laborales que se abren de forma creciente en el Estado chileno. Por otro lado, a la coexistencia del funcionalismo y el marxismo (Barrios, 1986:1) como modelos de elucubración sobre lo social.

En dicha trama el desempeño profesional se expresa como un acto público y publicable, sobre el límite que se impone cada cual: unos resguardados por las posiciones académicas y vinculados a las prácticas dentro de la política científica, y otros definiéndose desde las plazas laborales tecno-burocráticas y en la acción política partidaria ${ }^{1}$.

En la complejidad de estas formas de pensar y actuar en lo social, nosotros sostenemos que el debate sobre la dependencia no sólo vino a expresar la síntesis de las posibilidades de la acción colectiva de los practicantes de la disciplina (Barrios, 1986:20; Portes, 2001:126), sino que, además, fijó el hito del desenvolvimiento que hasta esa época había logrado la sociología latinoamericana.

Un ejemplo que define una de las cualidades del clima de época es el debate sobre el uso del ensayo en el quehacer sociológico, debido a que devela las tensiones en la metodología para usar las lógicas de análisis sobre lo social. En ese contexto se inscribe la lucha por el contenido de la categoría desarrollo, lo que particularmente se puede ver con el concepto de integración.

Por un lado, Germani (1971) supone que las dinámicas de la integración estarían dada por fases que funcionan como modelos que inciden en las sociedades en transición, mientras que en la propuesta del análisis integrado de Cardoso y Faletto (2003), la noción de fase es una herramienta heurística para nombrar las

1 "Así, la idea de neutralidad valorativa de la ciencia que había precedido a la profesionalización de la disciplina es ahora abandonada, siendo reemplazada por la noción de compromiso valorativo, que opone ciencia académica (o burguesa) a ciencia comprometida o militante. La vocación del científico y del político tiende a fundirse en la imagen del sociólogo crítico de la realidad, como intelectual revolucionario o como transformador de la sociedad." (Brunner, 1988:237) 
dinámicas de las relaciones entre grupos que construyen históricamente la dependencia, con lo cual reubican la utilidad que observa Germani en cuanto a que la fase explica el proceso de diferenciación que acontece en la medida que una transición se realiza observando las experiencias de las economías capitalistas originarias.

Así el clima de la época anima a cada sociólogo a identificar antecedentes empíricos de los procesos de transición y, en esta gesta, la formulación de supuestos varía, mostrándonos un tránsito en el funcionamiento interno de la Sociología que se desplaza hacia la teoría de alcance intermedio, que busca ser un punto equidistante entre las respuestas que surgen habitualmente en el trabajo científico, y en las especulaciones que parten de una arquitectura general de la que se espera extraer regularidades accesibles al observador (Martín, 2000:32).

Para Portes (2001), el desplazamiento en el uso de las concepciones teóricas se puede comprender si asumimos que la Sociología ya estaba sustituyendo una lógica teórica deductiva por otra empírica inductiva, mediante lo cual los practicantes de la disciplina se sorprenden con los "acontecimientos del mundo real", impidiendo el ejercicio de predicción al cual estaba habituado el "teórico totalizante" (Portes, 2001:127-128).

En este contexto se debe entender la categoría de grupo (Germani, 1971:40; Cardoso y Faletto, 2003:13), debido a que ahí se juega la observación de la integración por ajuste y la integración valorativa. En la medida que, siguiendo a Germani (1971:63), se promueve la vinculación entre hipótesis "concretas a verificar".

Esta breve descripción de un ámbito del debate sobre la dependencia nos permite sostener que, hasta ese punto de la trayectoria, la Sociología ya había logrado la integración social de sus practicantes gracias a los instrumentos de conocimiento y de comunicación (Bourdieu, 2006:67) que se expresan como sus posesiones simbólicas. 
En consecuencia, su legitimación había sido lograda no sólo por ese "cuerpo de especialistas en un campo de producción y de circulación relativamente autónomo" (Bourdieu, 2006:70), sino porque estaban desplegadas las concurrencias por el monopolio de las competencias que sostienen el poder simbólico (Bourdieu, 2006:69) que facilita el representar a otros sectores sociales en la densidad de las plazas laborales propiamente científicas y en las propiamente políticas².

Con Brunner (1988:234-237) logramos observar que esta etapa de la sociología dentro del subcampo disciplinario de las universidades y de la academia, presenta tres cualidades y una consecuencia.

La primera cualidad se relaciona con un mercado de posiciones académicas más amplio y complejo que contribuye a la especialización de la Sociología. La segunda cualidad se refiere a una jerarquía institucional en el campo de las Ciencias Sociales. La tercera cualidad se vincula al surgimiento de una estructura de comunicación académica en el subcampo.

Luego, la consecuencia es el crecimiento de la cantidad de receptores de las producciones, o, como le nombra Brunner: "el efecto de difusión de la sociología" (1988:236) que se radicó en la elite política e ideológica del país.

En este sentido, y siguiendo la reflexión de Lechner (1988), esta etapa puede ser interpretada como el logro de una autonomía de los mercados de bienes simbólicos gracias a que la sociedad se abre a la diferenciación, lo cual promueve la formación de organizaciones que "certifican como legítimos" (Lechner, 1988:5) a ciertos productores de bienes simbólicos que, para la fecha, se trataría del trabajador que oficia de intelectual y que es reconoci-

2 Beigel (2010:16-17) presenta esta parte de la trayectoria de la Sociología chilena y argentina, tomando en cuenta tres funciones de la autonomía. Nosotros destacamos la segunda función, la Illusio, porque al retomar a Bourdieu, Beigel nos remarca la delimitación existente hasta ese trayecto en la sociología, y su consecuente capital simbólico sostenido en la dependencia entre dominantes y dominadores sobre la base de la estima, el reconocimiento, la fe, el crédito y el reconocimiento en los demás. 
do como el experto (Touraine, 1966:21; Garretón, 1989:5), el que administra el contenido de la plaza laboral en el Estado y que es nombrado como el tecnócrata, y el ideólogo militante (Garretón, 1989:5) que logra su legitimidad en la plaza laboral que el partido político le permite en el terreno de las razones plausibles o "endóxicas" (Bourdieu, 2006:114).

Desde los planteamientos de Micelli (2001), lo expuesto hasta aquí puede ser entendido por la dinámica de dos movimientos: uno radicado en el despliegue de las clases oligárquicas para crear un mercado laboral y otro anidado en las capacidades de los expertos, tecnócratas e ideólogos militantes para movilizar sus relaciones familiares y obtener plazas laborales en el Estado, las casas editoriales, los medios de comunicación y las universidades.

Posteriormente, y a medida que se abren diversos centros académicos y de investigación, aumenta la cantidad de núcleos y grupos que debaten ideas de las Ciencias Sociales (Brunner, 1985:10), se diversifican las plazas ofrecidas por los antiguos actores económicos, se amplía el mercado de diplomas que certifican los méritos profesionales, crece el consumo de literatura especializada y se forman nuevas expresiones políticas; el capital de relaciones sociales pierde peso en su cometido de reproducción directa de los productores de bienes simbólicos (Micelli, 2001:31-36) y se consolidan, asumiendo lo que propone Brunner (1985:10) para el sector "académico informal", los mecanismos sociales de identificación de la autoridad científica (Bourdieu, 2006:81-82) creados por las comunidades académicas regionales, indicadas, por ejemplo: en el tipo de participación en las escasas revistas especializadas de América y en la obtención de becas internacionales.

Desde esta perspectiva hay tres tipos de sociólogos legitimados, a saber: a) los que han movilizado su capital de relaciones sociales para acceder a las materias primas que organizan sus proyecciones y que les aseguran plazas laborales al interior de las 
organizaciones que amplían la autonomía del mercado de bienes simbólicos, b) los que han accedido a las plazas laborales por haber obtenido títulos que les reconocen sus capacidades técnicas y capitales escolares, y c) los que han usado un deteriorado capital de relaciones sociales y plazas laborales consagradas a la militancia en una o varias organizaciones, donde cabe una especial mención a la adscripción a un credo religioso y su reflejo en la militancia política.

\section{1. b. El período de la desposesión simbólica de la Sociología chilena}

A partir de 1973, el contenido de la plaza laboral del sociólogo experimenta una variación porque cambia la orientación del Estado chileno, el cual pone en funcionamiento un proceso de "desposesión simbólica" (Wacquant, 2007:129) de la sociología al interior del mercado de posiciones académicas y del tecno-burocrático ${ }^{3}$.

Para unos, como Garretón (1989), esto es un reemplazo de la Sociología por la Economía de origen norteamericano, la cual cumpliría el papel -así como lo hizo la Sociología- de formar a profesionales para las plazas laborales que ofrecerá el Estado y las empresas que ahora se relacionarán en el marco de una agenda neoliberal, la cual se caracterizará por dejar fuera de las competencias del Estado la administración de lo público y por ceder a las empresas la implementación de los servicios que resuelven los problemas colectivos, entre ellos: investigación y planificación para el desarrollo.

En este nuevo contexto socio-político, pareciera consolidarse un nuevo modelo de desarrollo de las Ciencias Sociales, ya prefigurado en el período anterior y cuyas características pueden definirse así. En primer lugar, en

3 Beigel (2010:23) nombra a esta etapa como un "proceso de reestructuración y desinstitucionalización". Estas nociones no contradicen la de desposesión simbólica, sino que ponen el énfasis en sus niveles complementarios como, por ejemplo: trayectoria de los estudiantes de postgrado, configuración de las agencias de investigación, procedencia de los criterios de acreditación y certificación. 
el plano universitario, la ley de universidades consagra un deterioro o degradación de las Ciencias Sociales, en la medida que sólo una de ellas es definida como de carácter exclusivamente universitario, la Economía. Las otras pueden ser enseñadas por instituciones no universitarias (...) El desarrollo disciplinario en las universidades se resiente fuertemente, así como la formación de nuevos científicos sociales. Más ligados a una cuestión presupuestaria que de orientación académica, empiezan a proliferar las enseñanzas de post-grado, especialmente en referencia a la Economía (Garretón, 1989:14-15).

Para otras, como Barrios, se trataría de la abdicación del funcionalismo que cede el paso a la Economía porque ella proporciona herramientas para explicar los hechos, permitiendo una "reacción tecnocrática frente a la excesiva ideologización previa" (Barrios, 1986:27).

Este periodo de desposesión simbólica de la Sociología por parte de la dictadura militar (1973-1989) nos muestra, por un lado, la dependencia de las relaciones que instituyen la plaza laboral para sociólogos a las variables de tipo de régimen político, especialmente a la forma que adoptan las disputas de las clases dirigentes por obtener el control del Estado, y al clima ideológico-político (Garretón, 1989:1).

A modo de ilustración, presentamos el comentario hecho en una entrevista:

Yo creo que el sociólogo pasó por una etapa de profeta, ahora tiene conciencia que sólo es un cientista social. Entonces creo que eso hace un nuevo tipo de afirmación y eso se expresa muy bien cuando se les ocurre hacer un Colegio de Sociólogos, o sea, hay un sentido profesional de pertenencia a una misma cosa en cuanto sociólogos, porque antes el sociólogo era primero marxista o democratacristiano o liberal, no sé que cosa, por lo menos en Chile era sí. En cambio ahora no, ahora es sociólogo pri- 
mero y después tiene sus particulares enfoques ideológicos y políticos (Entrevista AA-2, en Barrios, 1986:35).

Por otro lado, la desposesión de la sociología muestra que el poder simbólico logrado no es destruido, sino que hubo un reemplazo en su contenido, debido a lo cual las derivadas de la Economía: Ingeniería y Administración de Empresas, tienen a su disposición los mecanismos y artefactos socio-técnicos que permiten "hacer ver y de hacer creer, de confirmar o de transformar la visión del mundo y, por ello, la acción sobre el mundo, por lo tanto el mundo" (Bourdieu, 2006: 71). En la medida que su modo de enunciación constituye lo dado en los modelos de investigación, en las prácticas de transmisión de los saberes y en las plazas laborales de los practicantes.

A fines de la década de los ochentas podemos sostener, según los resultados que Culagovski $(1985: 13,26)$ logra de los asistentes al Congreso de Sociología realizado en la ciudad de Concepción, que los sociólogos chilenos no sólo han constatado la alteración negativa de la enseñanza disciplinaria, la cual está acompañada por una reducción de las plazas laborales en el Estado y en las universidades, al tiempo que aumentan las ubicadas en centros no oficiales.

Además cada cual sabe que se han incorporado dos nuevos contenidos legítimos mas no legitimadores, que están referidos a la existencia social del individuo que posee el grado académico y el título profesional de sociólogo, a saber: ocuparse en plazas laborales fuera de la profesión y cesantía estructural.

La emergencia de estos contenidos y sus desenlaces prácticos orientarán las actividades de los sociólogos, dando paso a una nueva estructura ocupacional. Por un lado, los que se encuentran cesantes o trabajando en otras actividades, verán como posible la creación de organizaciones con o sin fines de lucro, mediante lo cual diversifican la composición social de la plaza laboral y el contenido denso de su desempeño profesional. 
Por otro lado, a partir de 1973 y hasta 1985, los sociólogos que tienen empleos como tales, no sólo trabajarán en el Estado y las universidades. Además, participarán activamente en el expansivo crecimiento de los centros de investigación independientes o también llamados informales, los cuales son financiados por la cooperación internacional y que contribuyen a que el sociólogo conozca los modelos, técnicas y herramientas de la educación, específicamente de la Educación Popular; y dependiendo de los recursos económicos que logra la organización que le brinda la plaza de laboral, transitará hacia la investigación (Barrios, 1986:100-102).

A modo de ilustración, presentamos el comentario hecho en una entrevista:

Por otra parte, yo diría que las ciencias sociales en Chile hayan pasado de ser ciencias de financiamiento público, a ciencias de financiamiento privado, es un hecho que marca una forma de inserción de las ciencias sociales chilenas en la institucionalidad mundial de las ciencias sociales, y ese es un hecho nuevo, en la medida que la universidad ha dejado de tener un rol protagónico en la producción de las ciencias sociales, producto, básicamente, de la posibilidad de ayuda internacional a las ciencias sociales extrauniversitarias; este es, a mi juicio, un hecho que marca una forma particular de vínculo institucional (Entrevista a v-8, en Barrios, 1986:173).

Las tres características de esta nueva estructura ocupacional pueden describirse como: a) en ellas es imposible llevar a cabo un estudio sociológico sistemático porque no es válido ese ejercicio como contenido de las funciones de la plaza laboral, debido a que la posición del sociólogo es evaluada por el rendimiento de su reflexión sociológica como aporte a la intervención en lo social.

b) El desempeño del sociólogo está guiado por la negociación de los criterios que van a definir al programa financiable por la organización de la cooperación internacional. En consecuencia, el 
contenido denso de la plaza laboral entronca con la información técnica de la administración de programas.

Es decir, supone atisbar énfasis de financiamiento posible y probable, los cuales se logran con mayor probabilidad si se participa en las reuniones en las cuales se conversa, cara a cara, con los representantes de las Agencias. Luego, es necesario animar a los equipos de trabajo para concentrarse en los tópicos que han sido definidos en esa negociación, e iniciar el intenso quehacer de la formulación del proyecto que, una vez financiado, se transformará en el programa.

A modo de ilustración, exponemos una reflexión hecha en una entrevista:

El tema de la sociología con las agencias, o de los sociólogos con las agencias es un océano grande. Creo que la influencia no se da vía una simple imposición de tema, la comunidad de investigadores en Chile es lo suficientemente sofisticada como para estar allá en muy buen pie en las agencias. Las agencias contratan como consultores a cientistas sociales chilenos (...) porque esos puntos han salido de una interacción muy compleja y rica entre las agencias y los investigadores chilenos. (...) Además que el mismo mundo de las agencias que se relacionan con Chile, sus agentes, las personas, muchas veces están ideológicamente muy cercanas del sur, todo lo cual empujaría hacia el lado del argumento de que éste es el mejor de los mundos y hay poca dependencia, y que, en el fondo, nosotros estamos influyendo y determinando nuestras propias agendas de investigación, sólo que son financiadas por el norte (Entrevista a i-7, en Barrios, 1986:170).

Finalmente, c) en la plaza laboral el sociólogo deberá administrar el vínculo entre la conjetura y el saber práctico. Por lo cual, se sitúa en la metodología de la investigación-acción, debido a que este modo de producción le permite cumplir con los reque- 
rimientos de las organizaciones de la cooperación internacional que sustentan su plaza laboral.

A continuación, una opinión ilustra las características ya mencionadas.

La proliferación de los centros de estudio alternativos está vinculada a grupos políticos o religiosos y cada uno de ellos va constituyendo un espacio a partir de los proyectos que puede generar y de las fuentes financieras a las que puede llegar, es decir, yo veo que hay una estrecha relación entre los temas abordados por la sociología en esos centros y las fuentes de financiamiento; inclusive tengo la impresión de que cierta acumulación especializada de datos se debe, fundamentalmente, al origen del financiamiento de los proyectos, o sea, no al desarrollo de la especialización al interior de la sociología (...) sino más bien, de una especialización que espontáneamente se fue produciendo de acuerdo a la fuente de financiamiento (...) es decir, hay una serie de financiamiento especializado que conducen a sociologías especializadas (Entrevista a a-10, en Barrios, 1986:163-164).

En resumen, entre 1973 y 1989, el sociólogo tiene ante sí dos posibilidades para ejercer su disciplina. Por un lado, un ámbito del mercado formal constituido por el Estado, universidad y organizaciones sin fines de lucro y, por otro lado, un mercado laboral en construcción donde destacan las organizaciones sin fines de lucro que se mantienen gracias al financiamiento de la cooperación internacional y, en menor medida, las organizaciones con fines de lucro que brindan servicios a las empresas que se encuentran en transformación a raíz del giro del modelo económico implementado a partir de mediado de la década de los ochentas.

En este escenario el sociólogo sabe que hay una identidad profesional ligada a la relación Estado universidad pero, a diferencia de lo que sucedía en la década de los sesentas, también sabe que es el mercado quien dictaminará los criterios para que 
se desplieguen las razones prácticas que ayudan a elucubrar las soluciones a los problemas sociales, o para definir el vínculo que instituye la calidad de las plazas laborales.

De forma concomitante, en los sociólogos que se fueron formando en este período se observa un énfasis por dejar atrás las interpretaciones en "función de grandes paradigmas" (Barrios, 1986:45) para abrirse a la búsqueda de las especificidades nacionales que se manifiestan en: movimientos sociales, efectos "microsociales del autoritarismo" (Barrios, 1986:78), transición a la democracia, "concertación social" y en el estudio de los mecanismos de consenso (Barrios, 1986:83).

Debido a lo cual sucede la inauguración de los estudios sectoriales dedicados a develar las lógicas internas de estos objetos de investigación para atisbar nuevas categorías de interpretación y de sentidos políticos. Los que a diferencia del período anterior, donde se utilizó la idea de lo popular para conocer la acción política desplegada más allá del Estado y de los partidos políticos, se emplean las nociones de: sociedad civil, juventud, comunicación, mujer, marginalidad, cultura, cultura popular y minorías étnicas.

Y la teoría de alcance intermedio de Merton será el punto común de habla sobre lo social, y contribuirá a ser el marco donde se articule la legitimación en las plazas laborales que se ofrecen, las que se comienzan a caracterizar por la relevancia del quehacer metodológico, especialmente de los saberes sociales y técnicos para usar la investigación-acción, etnometodología y monografía; por la crítica frente al antiempirismo de la etapa 1960 a 1973, y porque se recurre a la historia para organizar un relato de lo social.

Para cerrar el resumen, a continuación exponemos dos opiniones que muestran las cualidades antes indicadas.

Opinión A1:

En ese sentido creo que hay un esfuerzo por hacer patente la realidad, de abordar problemas de forma lúcida, 
racional, no ya en el plano puramente filosófico, especulativo y menos todavía puramente en el plano exegético de los clásicos, sino en el plano epistemológico y metodológico, entendiendo la metodología como recurso de la epistemología, pero orientada a trasladar el discurso filosófico al plano de la construcción del conocimiento concreto (Entrevista z-8, en Barrios, 1986: 112).

Opinión A2:

Yo creo que el arrimarse a la historia tiene que ver con este fenómeno de relativizar las cosas, de no tener respuestas globales para todo; lo mismo ocurre con el empiricismo, por uno u otro lado, se empiezan a buscar acontecimientos concretos, específicos a nuestra realidad. Creo que ambos fenómenos, historia y empirismo, son parte de un esfuerzo de relativización, de una conciencia de lo relativos que son los modelos, las ciencias y, en general, las respuestas a las cosas humanas; esa percepción hace ir buscando en lo empírico y en lo histórico, ciertas señales (Entrevista aa-4, en Barrios, 1986:119).

\section{Conclusiones}

\subsection{Los contenidos del trabajo de los sociólogos a inicios de los noventas}

Según Culagovski (1985), a fines de la década de los ochentas los sociólogos chilenos pueden diferenciarse en dos grupos típicos por el "efecto generacional", cuyo contenido es la "experiencia de ruptura" provocada porque les "tocó (...) 1973" (Culagovski, 1985:10-11).

A nuestro entender, el contenido de la sociabilidad que distingue a los sociólogos deja en un tercer o cuarto nivel de relevancia a la variable edad, y al mismo tiempo, ubica en un nivel importante a: los hitos que atan a los contenidos de la sociabilidad de los sociólogos a las narraciones que legitiman su obrar en 
el Mercado de Trabajo, y a la construcción y reproducción de los mecanismos de legitimación que ellos prevén con el retorno a la democracia del sistema político chileno.

Desde una perspectiva complementaria, Barrios (1986:217) supone que en ese periodo de la trayectoria de la sociología chilena se consolida una comunidad profesional y no una comunidad científica, porque los sociólogos se referirán a sí mismos desde la legitimidad de la relación con los "colegas de trabajo" y gracias a "las experiencias personales de trabajo", dotando a la identidad profesional con los contenidos del saber tácito del desempeño en la plaza laboral (Culagovski, 1985:10).

Entonces, la década de los noventas volverá a expresar con claridad que el régimen político, ahora neoliberal, determina la trayectoria del Mercado Laboral. Ahora: amplio, diverso y difuso, y que a los contenidos de esas plazas les es común la "dimensión de la experiencia colectiva de la disciplina" (Barrios, 1986: xii). Es decir, la tensión constante entre la práctica científica y la intervención política. Luego, la orientación de los programas de investigación, la distribución del conocimiento y la reproducción de los practicantes de la disciplina, se verán condicionados por los contenidos de las plazas laborales.

Dicha impronta disciplinaria se vería instituida, como bien interpreta Barrios (1986: ii), gracias a las redes que facilitan que las carreras profesionales se construyan desde plazas laborales con contenidos disímiles: algunas responderían a intercambios, patrocinios y mutuas asistencias gobernadas por las calificaciones públicamente reconocidas y por honrar los estándares de la Sociología, mientras otras adoptarán contenidos distintos e incluso opuestos a las posesiones simbólicas de esas redes.

Lo excepcional en esta estructura del Mercado de Trabajo del sociólogo, es que un individuo puede lograr configurar su trayectoria participando en distintas plazas laborales, incluso en el mismo día. En consecuencia, la gestión de la identidad profesional no es transportada, sino que es asumida por el individuo en 
cada lugar de desempeño, debido a que es el contenido de las relaciones el que fija los criterios de la legitimidad del desempeño de sus funciones como sociólogo, y las expectativas que de él todos tienen.

Entonces es posible lograr dos posibilidades para interpretar el escenario donde se despliega la identidad del sociólogo hasta nuestros días. La primera es la de las motivaciones del sociólogo, y Culagovski las asume como la coexistencia de la orientación por "lo político", refiriéndose a la "sociología comprometida con el cambio social", y otra orientada por la academia y la ciencia, ligada al "esfuerzo intelectual por explicar lo social" (Culagovski, 1985:8). Desde ahí, la motivación se relaciona con tópicos, a saber: educación, política, familia, cultura, trabajo, salud, económica y metodología.

La segunda posibilidad está organizada desde el desempeño en la plaza laboral del sociólogo, donde se reconocen especialidades: a. El sociólogo como gerente abocado al marketing, b. El sociólogo empresario y administrador que va aprendiendo gestión, c. El sociólogo que sondea las tendencias y las convierte en hechos noticiosos que le interesan a los medios de comunicación, o, “empresario de la sociología" (Barrios, 1986:135), d. El sociólogo que elabora diagnósticos de organizaciones, o "asistente social de grupos" (Barrios, 1986:136), e. El sociólogo que se dedica a publicar, f. El sociólogo que trabaja en el sector público: Estado y municipalidades, g. El sociólogo que mantiene su plaza laboral gracias al financiamiento externo (Barrios, 1986:140), h. El sociólogo que trabaja en organizaciones no gubernamentales, donde diseña y administra programas sociales, mezclando acción y análisis social.

\subsection{El desafío de construir Ciencias Sociales autónomas en el Sur}

Las ideas que a continuación se plantean surgieron gracias al trabajo en el II Workshop, especialmente cuando lo que ya se ha leído fue puesto en común y, quien escribe, puso en cuestión al- 
gunos de esos planteamientos a raíz de las exposiciones de los doctores Yvez y Kreimer.

El primero nos propuso que la Sociología vino a nombrar y a ocupar la relación de mediación que existía entre la ciencia del gobierno y la ciencia del Estado. Luego, yo recojo los postulados del Dr. Kreimer desde mi interpretación, y supongo que la Sociología es un artefacto de la trayectoria de esa relación existente.

Si lo planteado es adecuado a la lógica de uso de esas categorías, resulta que los practicantes de la Sociología deben saber gestionar la traducción entre el gobierno de lo social y el gobierno del Estado y, particularmente, necesitan construir y reproducir dispositivos: técnicas de relevo de información, de construcción de datos, de análisis y de presentación de resultados, para tener la autoridad de representar porque esta es la carta eficiente de los productores de bienes simbólicos cuando hay procesos de autonomía del y en el campo científico.

Luego podríamos sostener que, ante la ausencia de esos dispositivos en un Mercado de Trabajo, los practicantes de la disciplina transformaron su capital social en capital científico. En ese desempeño, ellos lograron habitar las plazas del Estado porque los no sociólogos, supusieron que la Sociología tenía las herramientas para gobernar lo social, o, como hemos visto hasta 1973, expresaba conjeturas plausibles.

Entonces, los practicantes de la Sociología chilena fueron instituyendo relaciones que reunieron funciones reconocidas como propiamente sociológicas, o, la plaza laboral en el mercado de posiciones académicas y en el tecno-burocrático; crearon y consolidaron audiencias: élite política e intelectual, y lograron conformar un sistema de códigos socioculturales que ordenó lo social, por ejemplo: modernización, desarrollo, grupos, dependencia. Entonces, gobernaban lo social. Y esos códigos ayudaron a intervenir en él, por ejemplo: planificación, proyecto, programa. Entonces, lo dominaban. Ambos aspectos conformaron el capital simbólico del desempeño de esa trayectoria. 
A continuación, el Golpe de Estado del 11 de septiembre de 1973, que es una "acción faccionalista" (Grez y Salazar, 1999: 10) que representa una "operación estratégica" en el marco de una "revolución neoliberal" (Salazar, 2006: 96), abrió el proceso de "desposesión simbólica" (Wacquant, 2007:129) de la Sociología gracias a la destrucción de sus fuentes de legitimación, y permitió reconstruir un nuevo sistema de relaciones donde la Economía ocupó tanto la posición en la trayectoria de la relación entre la ciencia del gobierno y la ciencia del Estado, como el capital simbólico logrado por la autonomía del campo científico y tecnoburocrático, demostrando que la autoridad que permite representar lo social: gobernarlo y dominarlo, no era propiedad exclusiva de la Sociología.

Luego y con especial relevancia en la década de los ochentas, la plaza laboral del sociólogo se vuelve diversa y su contenido difuso, en la medida que hay un sector burocrático y académico reducido, y una expansión de lugares de ejercicio en las organizaciones no gubernamentales. Posteriormente, en la década de los noventas, el contenido denso de la plaza laboral para los sociólogos se ve definido por el desempeño de la Economía. Por tanto, existe un consenso epistemológico que entrega los criterios que definen el diálogo y la actividad mediadora entre la ciencia del gobierno y la ciencia del Estado, a lo que regularmente se le nombra como "el modelo neoliberal", y cuya expresión de realidad es el mercado y las posibilidades de verdad están adecuadas a las necesidades de los consumidores de los bienes simbólicos.

Entonces, y como ya lo señalamos en la primera conclusión de este documento, la gestión de la identidad profesional no es transportada, sino que es asumida por el individuo en cada lugar de desempeño. Y, según el trazo de esta última conclusión, la sociología comprometida con el cambio social deja de ser una carta eficiente para los mecanismos de integración del sociólogo al Mercado de Trabajo, arrastrando consigo al esfuerzo intelectual por explicar lo social. Porque, en esta nueva etapa de la 
trayectoria de la Sociología, serán las cualidades de la plaza laboral ofrecida a los sociólogos, las que definan la integración y la identidad profesional como: gerente de marketing, empresario y emprendedor, asistente social de grupos, diseñador de proyectos o metodólogo que brinda servicios de evaluación de impacto de las políticas públicas o de los programas de responsabilidad social de las organizaciones.

\section{Bibliografía}

Barrios, Alicia, 1986, La construcción social de una disciplina: El caso de la Sociología en Chile, FLACSO-Chile.

Beigel, Fernanda, 2010, "Reflexiones sobre el uso del concepto de campo y acerca de la "elasticidad" de la autonomía en circuitos académicos periféricos", en Beigel, Fernanda (comp), 2010, Autonomía y dependencia académica. Universidad e investigación científica en un circuito periférico: Chile y Argentina (19501980), pp. 13-46, Buenos Aires, Ediciones Biblos.

Brunner, José, 1988, El modo de hacer sociología en Chile (p. 221-, en Barrios, Alicia, y Brunner, José, 1988, La Sociología en Chile, Instituciones y Practicantes, Santiago, FLACSO-Chile.

Bourdieu, Pierre, 1996, Intelectuales, Política y Poder, Buenos Aires, Ediciones Eudeba.

Brunner, José, 1985, La participación de los centros académicos privados en el desarrollo de las Ciencias Sociales, Santiago de Chile, Documento de trabajo núm. 257, Programa FLACSO-Chile.

Cardoso, Fernando, y Faletto, Enzo, 2003, Dependencia y Desarrollo en América Latina. Ensayo de interpretación sociológica, Buenos Aires, Ediciones Siglo Veintiuno.

Culagovski, Mauricio, 1985, Estudios del Campo Científico VII. El Profesional Sociólogo en Chile: Resultados preliminares de una encuesta, FLACSO-Chile.

Garretón, Manuel, 1989, La Evolución de las Ciencias Sociales en Chile y su Internalización. Una Síntesis, Chile, Documento de trabajo núm. 432, Programa FLACSO-Chile.

Germani, Gino, 1971, Política y Sociedad en una época de transición, Buenos Aires, Ediciones Paidós.

Gómez, Justino y Sandoval, Mario, 2004, más allá del oficio del sociólogo: 
Nuevas identidades, prácticas y competencias en el campo profesional, Santiago, Ediciones Universidad Católica Silva Henríquez.

Grez, Sergio y Salazar, Gabriel (com), 1999, Manifiesto de Historiadores. Santiago, Lom.

Kuhn, Tomás, 1992, La estructura de las revoluciones científicas, Santa fe de Bogotá, Ediciones Fondo de Cultura Económica.

Martín, Oliver, 2000, Sociología de la Ciencia, Buenos Aires, Ediciones Nueva Visión.

Micelli, Sergio, 2001, Intelectuais á brasileira, San Pablo, Ediciones Companhia Das Letras.

Lechner, Norbet, 1988, Los desafíos de las Ciencias Sociales en América Latina, Santiago, Documento de Trabajo Programa FLACSOChile, núm. 372.

Portes, Alejandro, 2001, La sociología en el Hemisferio. Hacia una agenda conceptual, en número 178 de la Revista Nueva Sociedad (pp. 126-144).

Salazar, Gabriel, 2006, Ser niño "huacho" en la historia de Chile (siglo XIX), Santiago, LOM.

Touraine, Alain, 1966, "Unidad y diversidad de la sociología”, en Ciencias Sociales: Ideología y realidad nacional. Buenos Aires, Ediciones Tiempo Contemporáneo.

Wacquant, Loïc, 2007, Parias Urbanos. Marginalidad en la ciudad a comienzo del milenio, Buenos Aires, Ediciones Manantial. 